\title{
Screening potential microRNAs associated with pancreatic cancer: Data mining based on RNA sequencing and microarrays
}

\author{
JING MA ${ }^{1 *}$, SIWEN SUN $^{1 *}$, CHEN SONG $^{1}$, NING LI $^{2}$, NA LI $^{1}$, LINGZHI XU ${ }^{1}$, \\ TING YANG ${ }^{3}$, YULONG LAN ${ }^{3}$ and MAN LI ${ }^{1}$ \\ ${ }^{1}$ Department of Oncology, The Second Affiliated Hospital of Dalian Medical University; \\ ${ }^{2}$ Department of Foreign Languages, Dalian Medical University; ${ }^{3}$ Department of Neurosurgery, \\ The Second Affiliated Hospital of Dalian Medical University, Dalian, Liaoning 116023, P.R. China
}

Received October 11, 2018; Accepted January 17, 2020

DOI: $10.3892 /$ etm.2020.8991

\begin{abstract}
Pancreatic cancer is a malignant tumor of the digestive tract, rendering it difficult to make an accurate diagnosis. The 5 year survival rate for pancreatic cancer is $<1 \%$, and surgical resection rarely proves to be effective. Therefore, the identification of more effective methods for the early detection of pancreatic cancer is an urgent requirement. The present study aimed to explore key genes and microRNAs (miRNAs) associated with the pathogenesis of pancreatic cancer. Public databases were searched, and the data were integrated from The Cancer Genome Atlas and Gene Expression Omnibus databases, leading to the identification of 23 differentially expressed miRNAs (DE-miRNAs). A total of four of the DE-miRNAs were upregulated (hsa-miR-892b, hsa-miR-194-2, hsa-miR-200a and hsa-miR-194-1), whereas 19 downregulated DE-miRNAs (hsa-miR-424, hsa-miR-191, hsa-miR-484, hsa-miR-142, hsa-miR-15b, hsa-miR-450a-1, hsa-miR-423, hsa-miR-126, hsa-miR-505, hsa-miR-16-1, hsa-miR-342, hsa-miR-130a, hsa-miR-3613, hsa-miR-450a-2, hsa-miR-26b, hsa-miR-451, hsa-miR-19b-2, hsa-miR-106a and hsa-miR-503) were identified using the cut-off criteria of $\mathrm{P}<0.05$ and $\mid \log 2 \mathrm{FCl}>1.0$.
\end{abstract}

Correspondence to: Professor Man Li, Department of Oncology, The Second Affiliated Hospital of Dalian Medical University, 467 Zhongshan Road, Dalian, Liaoning 116023, P.R. China

E-mail: dyeryliman@yeah.net

*Contributed equally

Abbreviations: miRNA, microRNAs; TCGA, The Cancer Genome Atlas; GEO, Gene Expression Omnibus; DE, differentially expressed; DEGs, differentially expressed genes; FC, fold change; KEGG, Kyoto Encyclopedia of Genes and Genomes; GO, Gene ontology; BP, biological process; OS, overall survival; HPA, Human Protein Atlas

Key words: pancreatic cancer, RNA sequencing, gene expression profile, differentially expressed microRNAs, target, treatment, bioinformatics analysis
Hsa-miR-3613-5p was identified as a prognostic DE-miRNA. The functional enrichment analyses demonstrated that the target genes of hsa-miR-3613-5p may be associated with the p53 signaling pathway. Survival analysis performed for genes in the p53 signaling pathway revealed that cyclin-dependent kinase 6 and ribonucleoside-diphosphate reductase subunit M2 may be the most likely to be associated with prognostic value. The integrated analysis performed in the current study demonstrated that hsa-miR-3613-5p may be used as a potential prognostic marker for pancreatic cancer.

\section{Introduction}

Pancreatic cancer is a malignancy of the pancreas, which is associated with a poor prognosis $(1,2)$, and the morbidity and mortality rates associated with pancreatic cancer have increased globally in recent years (3-5). Currently, surgery is the only possible cure for pancreatic cancer, however, due to the high degree of malignancy, surgical resection rates are low, with unsatisfactory treatment efficacies $(6,7)$. Therefore, the identification of a novel means for early diagnosis and effective treatment for pancreatic cancer is urgently required. A current lack of knowledge regarding the exact molecular mechanism(s) of pancreatic cancer progression reduces the possibility of early diagnosis and timely treatment $(8,9)$. Therefore, the identification of effective biomarkers to improve understanding of the possible pathogenesis of pancreatic cancer is an urgent requirement.

It is well established that microRNAs (miRNAs) are key components of the non-coding RNA family that are capable of extensively regulating gene expression (10-12). The mature miRNAs are derived from their precursors, either generated from spliceosomes or transcribed from the genome (13). Previously published studies have confirmed that miRNAs are abnormally expressed in a variety of malignancies, and function either as oncogenes or as tumor suppressors (14-16). miRNAs exhibit the characteristics of high tissue specificity and stability, with altered expression patterns during tumor development (17-19). Therefore, the potential of miRNAs in the diagnosis of a variety of cancer types may provide researchers with a means to identify and characterize potential biomarkers. 
In the present study, data were accessed from The Cancer Genome Atlas (TCGA) and Gene Expression Omnibus (GEO) databases to explore key miRNAs in pancreatic cancer. Comprehensive bioinformatics analysis was used to identify differentially expressed miRNAs (DE-miRNAs). The target genes of prognostic DE-miRNAs were predicted. Gene Ontology (GO) and Kyoto Encyclopedia of Genes and Genomes (KEGG) analyses were subsequently performed to analyze the functional enrichment of the gene set, and to identify significant pathways that are associated with the target genes. The present study aimed to investigate the involvement of miRNAs critical to pancreatic cancer progression, and to seek a means of improved disease diagnosis.

\section{Materials and methods}

Data processing and identification of DE-miRNAs. The miRNA sequencing data were downloaded from FireBrowse (version 0.4.13; http://firebrowse.org/), which is a data integration database associated with the TCGA database. The data were processed using $\mathrm{R}$ language (version 3.4.4; https://www.r-project.org/) and normalized by $\log _{2}$ transformation. miRNAs that were differentially expressed between pancreatic cancer and healthy tissues were analyzed using the Limma package in R language with the criteria of llog2FC (fold change) $>1.0$ and $\mathrm{P}<0.05$. Results are presented in volcano plots, which were plotted using GraphPad prism.v.5 (GraphPad Software, Inc.), and in heat maps using the open source markup language, HEML (20). The gene expression profile (GSE62452) and miRNA expression profile (GSE43796) $(21,22)$ were downloaded from the GEO database (https://www.ncbi. nlm.nih.gov/geo/). A total of 31 samples were identified in GSE43796, including 26 pancreatic cancer samples and 5 healthy samples. The GSE43796 data were collected based on the GPL15159 platform Agilent-031181 Unrestricted_Human miRNA_V16.0_Microarray 030840 (probe name version). The GSE62452 dataset containing 130 samples, including 69 tumor tissues and 61 adjacent tissues, were collected based on the GPL6244 platform (HuGene-1_0-st) Affymetrix Human Gene 1.0 ST Array [transcript (gene) version]. Profiles were analyzed using the GEO2R online tool (http://www.ncbi. nlm.nih.gov/geo/geo2r/).

Overall survival (OS) analysis and target gene prediction. OS analyses were evaluated using the online tool, OncoLnc (23), which links TCGA survival data to the expression of mRNA, miRNA or long non-coding RNA (lncRNA). miRNAs or genes were identified if they were significantly associated with OS. Target genes were predicted using the online analysis tools, TargetScan (http://www.targetscan.org/) (24) and DIANA-mT (http://diana.imis.athena-innovation.gr/DianaTools/index.php?r= mrmicrot/index) $(25,26)$. To further enhance the accuracy of the bioinformatics analysis, Venn diagrams were used to identify overlapping target genes.

Functional enrichment analyses. KEGG pathway and GO enrichment analyses were performed for the functional annotation and pathway analyses, to identify the biological processes, cellular components, molecular functions and biological pathways that the genes were significantly enriched in. The results were visualized using ClueGO, which is one of the apps in Cytoscape (27). A gene count $\geq 3$ was set as the cut-off criterion.

Gene expression comparison. The Human Protein Atlas (version 15.0; https://www.proteinatlas.org) (28), an open platform to provide large amounts of transcriptomics and proteomics data of all 24,000 human proteins, is composed of a Tissue Atlas, Cell Atlas, and Pathology Atlas. Immunohistochemistry results (https:// v15.proteinatlas. org/ENSG00000105810-CDK6/tissue/pancreas; https://v15. proteinatlas.org/ENSG00000105810-CDK6/cancer/tissue/pancreatic+ cancer; https://v15.proteinatlas.org/ENSG00000171848RRM2/tissue/pancreas; and https://v15.proteinatlas.org/ ENSG00000171848-RRM2/cancer/tissue/pancreatic+cancer) were obtained from this database to make comparisons between healthy pancreatic tissues and pancreatic tumor tissues.

\section{Results}

Overall presentation of the entire analytical process. The Limma package in $\mathrm{R}$ language was used to analyze the miRNA sequencing data from the TCGA database to identify the DE-miRNAs. Toincrease the accuracy of the results, two microarrays were downloaded from the GEO database during the analysis. These results demonstrated that hsa-miR-3613-5p may be the most reliable prognostic DE-miRNA. The target genes of hsa-miR-3613-5p were predominantly enriched in 20 KEGG pathways, and the p53 signaling pathway was the main focus in the final analysis. Furthermore, survival analysis of genes in the p53 signaling pathway was also performed, and only the genes for cyclin-dependent kinase 6 (CDK6) and ribonucleoside-diphosphate reductase subunit M2 (RRM2) exhibited prognostic value. A flow-chart of the entire analysis is presented in Fig. 1.

Identification of DE-miRNAs in miRNA sequencing data and OS analysis. According to the criteria of $\mid \log 2 \mathrm{FCl}>1.0$ and $\mathrm{P}<0.05$, a total of 23 DE-miRNAs in miRNA sequencing data were identified, including four upregulated (hsa-miR-892b, hsa-miR-194-2, hsa-miR-200a and hsa-miR-194-1) and 19 downregulated (hsa-miR-424, hsa-miR-191, hsa-miR-484, hsa-miR-142, hsa-miR-15b, hsa-miR-450a-1, hsa-miR-423, hsa-miR-126, hsa-miR-505, hsa-miR-16-1, hsa-miR-342, hsa-miR-130a, hsa-miR-3613, hsa-miR-450a-2, hsa-miR-26b, hsa-miR-451, hsa-miR-19b-2, hsa-miR-106a and hsa-miR-503) miRNAs (Table I). The results are also presented as a volcano plot and heat-map (Fig. 2). To identify the miRNAs that could potentially be associated with OS of patients with pancreatic cancer, the online tool OncoLnc was used to access TCGA survival data to evaluate the association between miRNA expression and patient survival. Two miRNAs were identified that may be associated with OS, with P-values and log-rank values of $\mathrm{P}<0.05$ (Fig. 3). These were hsa-miR-126-5p and hsa-miR-3613-5p, which are highlighted in the volcano plot (Fig. 2).

Target gene prediction. Subsequently, datasets associated with miRNA and pancreatic cancer were searched in 


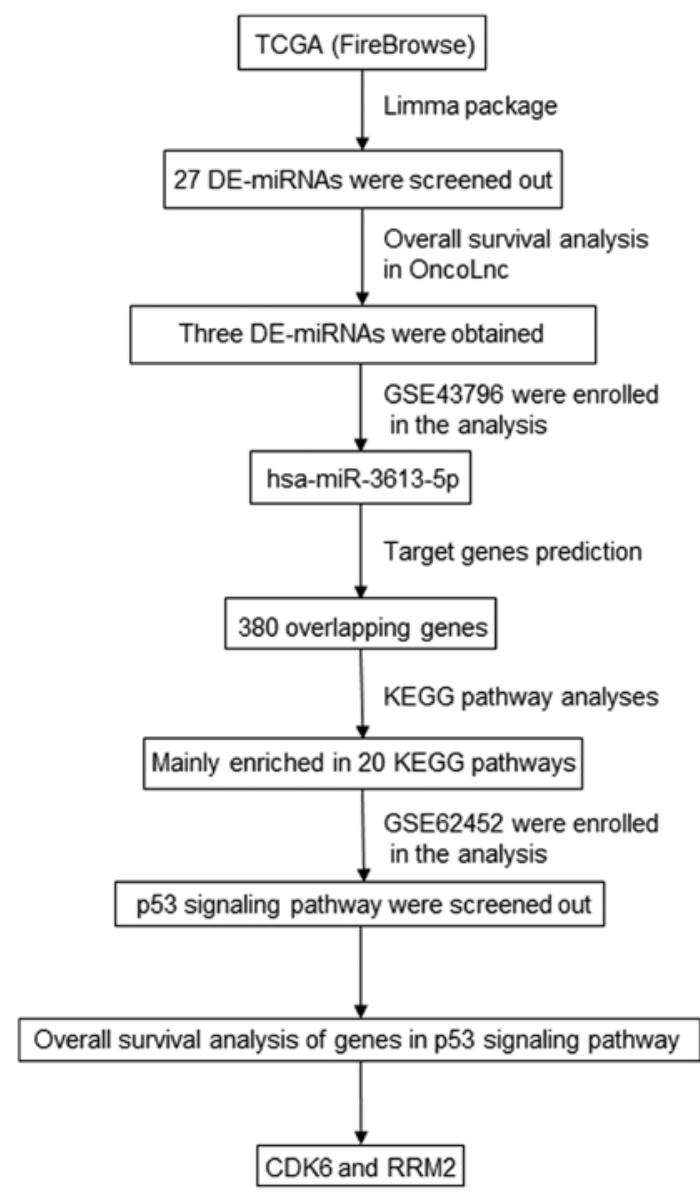

Figure 1. Flow chart of the entire article analysis. The present study comprised several procedures; namely GEO-based verification, TCGA-based data aggregation of miRNA-seq, and multiple bioinformatics analyses. GEO, Gene Expression Omnibus; TCGA, The Cancer Genome Atlas; miRNA, microRNA; DE, differentially expressed; KEGG, Kyoto Encyclopedia of Genes and Genomes; CDK6, cyclin-dependent kinase 6; RRM2, ribonucleoside-diphosphate reductase subunit M2.

the GEO database, and the following data were selected: i) $>30$ samples; ii) non-coding RNA profiles; and iii) the period between January 1, 2013 and December 31, 2018. GSE43796 from the GEO database was accessed, and the GEO2R online tool was used to obtain the DE-miRNAs. The top 250 miRNAs were thereby identified (Table II), and the two above-mentioned prognostic miRNAs intersected, leaving only hsa-miR-3613-5p. The association between hsa-miR-3613 and clinical features was evaluated in patients with breast cancer (Table SI). The results indicated that hsa-miR-3613 was significantly associated with clinical stage $(\mathrm{P}=0.034)$ and histologic type $(\mathrm{P}=0.002)$. Target genes of hsa-miR-3613-5p were predicted using the TargetScan and DIANA-MT online analysis tools. As a result of this analysis, a total of 380 overlapping genes of hsa-miR-3613-5p were identified (Fig. 4).

KEGG pathway and GO enrichment analyses of target genes. To elucidate the biological function of overlapping genes, enrichment analyses were performed using ClueGO. The results obtained indicated that genes were predominantly enriched in $20 \mathrm{KEGG}$ pathways, including the tissue growth factor- $\beta$, p53 and glioma signaling pathways
Table I. Differentially expressed miRNAs in TCGA.

\begin{tabular}{lccc}
\hline A, Upregulated & & & \\
\hline miRNA_ID & $\log _{2}$ FC & P-value & adj.P.Val \\
\hline hsa-miR-892b & 1.530606 & $2.62 \times 10^{-5}$ & $5.48 \times 10^{-3}$ \\
hsa-miR-194-2 & 2.544289 & $2.89 \times 10^{-4}$ & $1.89 \times 10^{-2}$ \\
hsa-miR-200a & 2.161983 & $3.18 \times 10^{-4}$ & $1.92 \times 10^{-2}$ \\
hsa-miR-194-1 & 2.497915 & $3.98 \times 10^{-4}$ & $2.19 \times 10^{-2}$
\end{tabular}

B, Downregulated

\begin{tabular}{lllr}
\hline miRNA_ID & $\log _{2} \mathrm{FC}$ & P-value & \multicolumn{1}{c}{ adj.P.Val } \\
\hline hsa-miR-424 & -1.94731 & $1.00 \times 10^{-6}$ & $5.24 \times 10^{-4}$ \\
hsa-miR-191 & -1.29315 & $1.88 \times 10^{-5}$ & $4.91 \times 10^{-3}$ \\
hsa-miR-484 & -1.17521 & $5.37 \times 10^{-5}$ & $9.356 \times 10^{-3}$ \\
hsa-miR-142 & -2.36313 & $6.35 \times 10^{-5}$ & $9.49 \times 10^{-3}$ \\
hsa-miR-15b & -1.12279 & $8.34 \times 10^{-5}$ & $1.09 \times 10^{-2}$ \\
hsa-miR-450a-1 & -1.66695 & $1.05 \times 10^{-4}$ & $1.21 \times 10^{-2}$ \\
hsa-miR-423 & -1.12274 & $1.15 \times 10^{-4}$ & $1.21 \times 10^{-2}$ \\
hsa-miR-126 & -1.44387 & $1.90 \times 10^{-4}$ & $1.72 \times 10^{-2}$ \\
hsa-miR-505 & -1.24908 & $2.19 \times 10^{-4}$ & $1.76 \times 10^{-2}$ \\
hsa-miR-16-1 & -1.01975 & $2.46 \times 10^{-4}$ & $1.84 \times 10^{-2}$ \\
hsa-miR-342 & -1.50755 & $2.86 \times 10^{-4}$ & $1.89 \times 10^{-2}$ \\
hsa-miR-130a & -1.1491 & $3.30 \times 10^{-4}$ & $1.92 \times 10^{-2}$ \\
hsa-miR-3613 & -1.44412 & $5.13 \times 10^{-4}$ & $2.55 \times 10^{-2}$ \\
hsa-miR-450a-2 & -1.55496 & $5.65 \times 10^{-4}$ & $2.69 \times 10^{-2}$ \\
hsa-miR-26b & -1.1955 & $7.51 \times 10^{-4}$ & $3.41 \times 10^{-2}$ \\
hsa-miR-451 & -2.71252 & $8.47 \times 10^{-4}$ & $3.60 \times 10^{-2}$ \\
hsa-miR-19b-2 & -1.38624 & $1.01 \times 10^{-3}$ & $4.04 \times 10^{-2}$ \\
hsa-miR-106a & -1.4397 & $1.07 \times 10^{-3}$ & $4.15 \times 10^{-2}$ \\
hsa-miR-503 & -1.6143 & $1.36 \times 10^{-3}$ & $4.91 \times 10^{-2}$ \\
\hline
\end{tabular}

miR, microRNA; TCGA, TCGA, The Cancer Genome Atlas.

(Fig. 5A). The GO biological process terms were predominantly enriched in 'central nervous system development' (Fig. 5B). To increase the accuracy, one further microarray (GSE62452) from the GEO database was also accessed. The following screens were selected: i) $>30$ samples; ii) expression profiles; and iii) the period between January 1, 2013 and December 31, 2018. The GEO2R online tool was applied to explore the differentially expressed genes, and functional analyses of the top 250 genes (Table SII) were performed. The p53 signaling pathway was also identified in the KEGG pathway (Fig. 6).

Survival analysis and prognostic gene expression of the p53 signaling pathway. An OS analysis of the genes in the p53 signaling pathway was performed. The results obtained indicated that only the CDK6 and RRM2 genes were associated with OS (Fig. 7A). Subsequently, the Human Protein Atlas was queried to obtain the immunohistochemistry results of CDK6 and RRM2 gene expression in healthy and cancer 


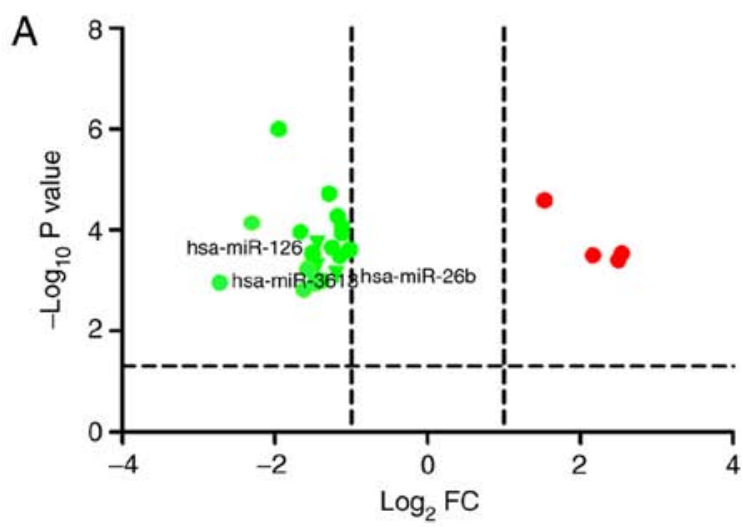

B

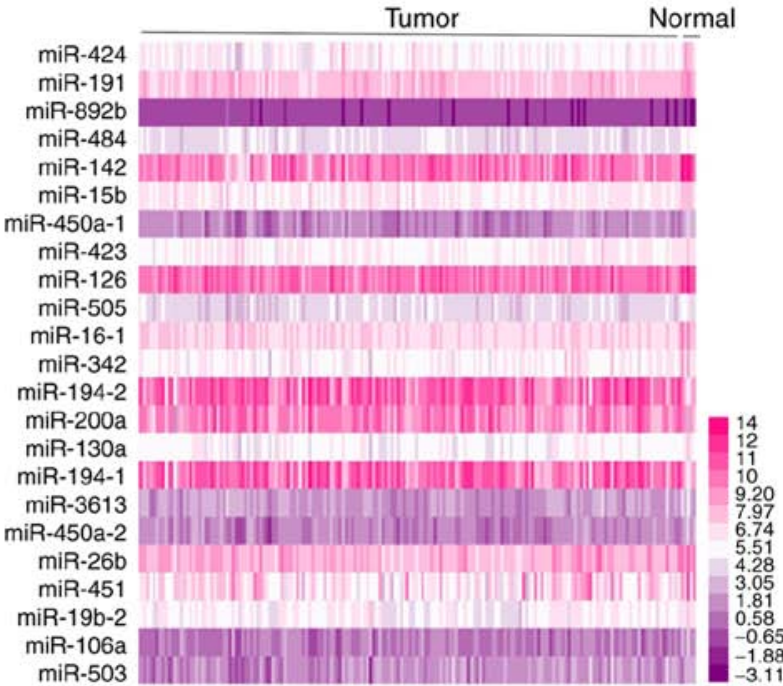

Figure 2. Volcano plot and heat-map of differentially expressed miRNAs. (A) Red dots indicated upregulated miRNAs, whereas the green dots indicated downregulated miRNAs. The cut-off criteria were $\log 2 \mathrm{FCl}>1.0$ and $\mathrm{P}<0.05$. Prognostic miRNAs are indicated in this Figure by the inverted triangles. (B) Heat-map of the differentially expressed miRNAs. According to the criteria of $\log 2 \mathrm{FCl}>1.0$ and $\mathrm{P}<0.05$, a total of $23 \mathrm{DE}-\mathrm{miRNAs}$ in miRNA sequencing data were identified, including 4 upregulated and 19 downregulated miRNAs. There are 183 samples, of which 179 are tumor and 4 are healthy. Pink coloration denotes miRNA upregulation, whereas purple indicates downregulation. miRNA, microRNA; DE, differentially expressed.

A

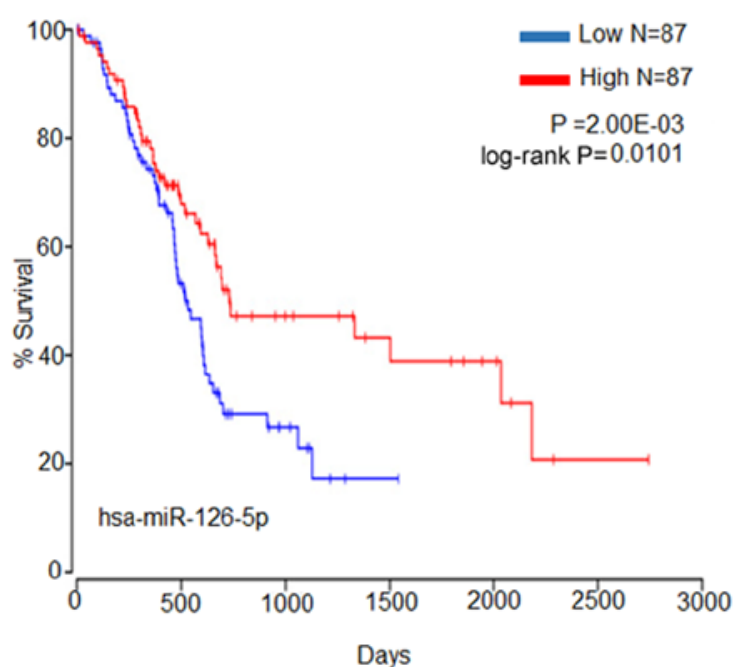

B

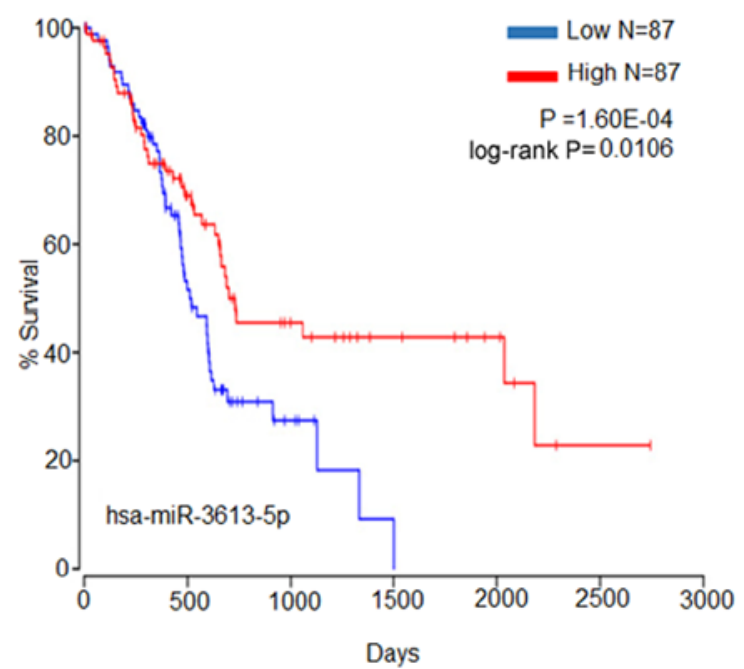

Figure 3. Overall survival analysis using the tool, OncoLnc. Three miRNAs were associated with overall survival in patients with pancreatic cancer. The patients were stratified into high-level and low-level groups. The Figure parts show (A) hsa-miR-126-5p and (B) hsa-miR-3613-5p. miRNA, microRNA.

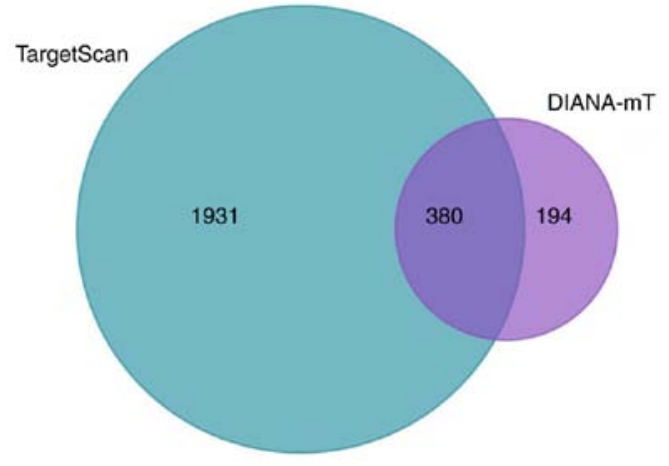

Figure 4. Target gene prediction for hsa-miR-3613-5p. The target genes were predicted using the TargetScan and DIANA-mT online analysis tools. miRNA, microRNA. tissues (Fig. 7B). All the results obtained were in agreement that the two genes are highly expressed in tumors, with poor prognosis.

\section{Discussion}

Pancreatic cancer is an aggressively malignant digestive tract tumor with a poor prognosis (29-31). The high mortality rate is due to the lack of early detection methods, and an inability to successfully treat patients once they are diagnosed (32). Understanding the molecular mechanism of pancreatic cancer progression is of critical importance to improve the diagnosis and treatment of this disease $(8,9,33)$. Currently, microarrays and high-throughput sequencing analyses have 
Table II. Top 250 miRNAs in the GSE43796 dataset.

\begin{tabular}{|c|c|c|c|c|c|c|c|}
\hline miRNA_ID & $\log _{2} \mathrm{FC}$ & P-value & adj.P.Val & miRNA_ID & $\log _{2} \mathrm{FC}$ & P-value & adj.P.Val \\
\hline hsa-miR-216b & -4.8039 & $4.65 \times 10^{-10}$ & $1.3 \times 10^{-6}$ & hsa-miR-4307 & -0.1549 & 0.000113 & 0.007447 \\
\hline hsa-miR-216a & -5.4037 & $8.97 \times 10^{-10}$ & $1.3 \times 10^{-6}$ & hsa-miR-613 & -0.1262 & 0.000114 & 0.007447 \\
\hline hsa-miR-216b & -3.716 & $1.10 \times 10^{-9}$ & $1.3 \times 10^{-6}$ & hsa-miR-148b & -0.1138 & 0.00013 & 0.008259 \\
\hline hsa-miR-216a & -4.8889 & $1.64 \times 10^{-9}$ & $1.44 \times 10^{-6}$ & hsa-miR-148a & -2.5367 & 0.000131 & 0.008259 \\
\hline hsa-miR-217 & -4.4452 & $2.34 \times 10^{-9}$ & $1.44 \times 10^{-6}$ & hsa-miR-3145-3p & -0.0763 & 0.000145 & 0.008957 \\
\hline hsa-miR-217 & -3.8 & $2.46 \times 10^{-9}$ & $1.44 \times 10^{-6}$ & hsa-miR-186 & 0.713 & 0.000164 & 0.009947 \\
\hline hsa-miR-216b & -3.169 & $3.30 \times 10^{-9}$ & $1.47 \times 10^{-6}$ & hsa-miR-302b & -0.1691 & 0.000185 & 0.011055 \\
\hline hsa-miR-216a & -4.0446 & $3.34 \times 10^{-9}$ & $1.47 \times 10^{-6}$ & hsa-miR-942 & -0.1017 & 0.000189 & 0.011107 \\
\hline hsa-miR-217 & -3.1523 & $1.26 \times 10^{-8}$ & $4.94 \times 10^{-6}$ & hsa-miR-99b ${ }^{a}$ & -0.1313 & 0.000207 & 0.011853 \\
\hline hsa-miR-216a & -1.6859 & $2.2 \times 10^{-7}$ & $7.75 \times 10^{-5}$ & hsa-miR-5481 & -0.0957 & 0.000212 & 0.011853 \\
\hline hsa-miR-216b & -0.4224 & $8.8 \times 10^{-7}$ & 0.000282 & hsa-miR-3681 & -0.0908 & 0.000212 & 0.011853 \\
\hline hsa-miR-4269 & -1.3437 & $1.64 \times 10^{-6}$ & 0.000483 & hsa-miR-93a & 0.1248 & 0.00022 & 0.011853 \\
\hline hsa-miR-217 & -1.2283 & $2.28 \times 10^{-6}$ & 0.000618 & hsa-miR-361-5p & 0.9421 & 0.000225 & 0.011853 \\
\hline hsa-miR-4269 & -1.4259 & $2.49 \times 10^{-6}$ & 0.000626 & hsa-miR-582-5p & 1.6334 & 0.000227 & 0.011853 \\
\hline kshv-miR-K12-8 & -1.568 & $3.17 \times 10^{-6}$ & 0.000744 & hsa-miR-3613-5p & -0.1532 & 0.000231 & 0.011853 \\
\hline hsa-miR-564 & -1.1939 & $4.87 \times 10^{-6}$ & 0.000963 & hsa-miR-3132 & -1.3333 & 0.000231 & 0.011853 \\
\hline hsa-miR-564 & -1.2361 & $5.06 \times 10^{-6}$ & 0.000963 & hsa-miR-553 & -0.1416 & 0.000232 & 0.011853 \\
\hline hsa-miR-3616-3p & -0.4127 & $5.14 \times 10^{-6}$ & 0.000963 & hsa-miR-302b & -0.1588 & 0.000239 & 0.011976 \\
\hline hsa-miR-130b ${ }^{a}$ & -0.3289 & $5.46 \times 10^{-6}$ & 0.000963 & hsa-miR-423-3p & 0.3666 & 0.000241 & 0.011976 \\
\hline hsa-miR-564 & -1.1964 & $5.72 \times 10^{-6}$ & 0.000963 & hcmv-miR-US25-1 ${ }^{\mathrm{a}}$ & -0.1146 & 0.000247 & 0.012062 \\
\hline kshv-miR-K12-8 & -1.4459 & $5.74 \times 10^{-6}$ & 0.000963 & hsa-miR-548t & -0.1388 & 0.000262 & 0.012307 \\
\hline hsa-miR-1307 & 0.5635 & $6.74 \times 10^{-6}$ & 0.001079 & hsa-miR-521 & -0.082 & 0.000262 & 0.012307 \\
\hline hsa-miR-3916 & -0.1009 & $8.93 \times 10^{-6}$ & 0.001368 & hsa-miR-3129-5p & -0.1044 & 0.000262 & 0.012307 \\
\hline hsa-miR-320e & 0.8174 & $9.66 \times 10^{-6}$ & 0.001419 & hsa-miR-99b & 1.5986 & 0.000267 & 0.012366 \\
\hline hsa-miR-944 & -0.1126 & $1.15 \times 10^{-5}$ & 0.001617 & hsa-miR-105 & -0.1008 & 0.000271 & 0.012399 \\
\hline hsa-miR-106b & 0.9524 & $1.39 \times 10^{-5}$ & 0.001887 & hsa-miR-3179 & -0.0771 & 0.000283 & 0.0128 \\
\hline hsa-miR-148a ${ }^{a}$ & -0.4605 & $1.6 \times 10^{-5}$ & 0.002029 & hsa-miR-186 & 0.6795 & 0.000309 & 0.013787 \\
\hline hsa-miR-130b & -1.9221 & $1.61 \times 10^{-5}$ & 0.002029 & hsa-miR-1306 & 0.234 & 0.000317 & 0.01397 \\
\hline hsa-miR-99b ${ }^{a}$ & -0.1775 & $1.88 \times 10^{-5}$ & 0.002286 & hsa-miR-1289 & -0.0883 & 0.000328 & 0.014269 \\
\hline hsa-miR-498 & 0.4483 & $1.97 \times 10^{-5}$ & 0.002315 & hsa-miR-579 & -0.1067 & 0.00034 & 0.014628 \\
\hline hsa-miR-148a $\mathrm{a}^{\mathrm{a}}$ & -1.2935 & $2.04 \times 10^{-5}$ & 0.002316 & hsa-miR-301a & 1.8527 & 0.00036 & 0.015276 \\
\hline hsa-miR-671-3p & 0.1226 & $2.17 \times 10^{-5}$ & 0.002391 & hsa-miR-148a & -2.337 & 0.00037 & 0.015288 \\
\hline hsa-miR-3164 & -0.1302 & $2.84 \times 10^{-5}$ & 0.002998 & hsa-miR-103a & 1.124 & 0.000371 & 0.015288 \\
\hline hsa-miR-548x_v16.0 & 0.1154 & $2.94 \times 10^{-5}$ & 0.002998 & ebv-miR-BART13 & -0.582 & 0.000373 & 0.015288 \\
\hline hsa-miR-106b & 1.1226 & $3.02 \times 10^{-5}$ & 0.002998 & hsa-miR-448 & -0.1176 & 0.000381 & 0.015434 \\
\hline hsa-miR-320d & 0.7308 & $3.06 \times 10^{-5}$ & 0.002998 & hsa-miR-299-5p & -1.0644 & 0.000401 & 0.015917 \\
\hline hsa-miR-4299 & -1.905 & $3.8 \times 10^{-5}$ & 0.003576 & hsa-miR-582-5p & 1.5887 & 0.000402 & 0.015917 \\
\hline hsa-miR-548o & -0.118 & $3.86 \times 10^{-5}$ & 0.003576 & hsa-miR-634 & 0.1183 & 0.000431 & 0.016744 \\
\hline hsa-miR-93 & 1.2234 & $4 \times 10^{-5}$ & 0.003611 & hsa-miR-3175 & -0.054 & 0.000433 & 0.016744 \\
\hline hsa-miR-331-3p & 1.1039 & $4.39 \times 10^{-5}$ & 0.003864 & hsa-miR-148a $a^{a}$ & -0.1302 & 0.00044 & 0.016859 \\
\hline hsa-miR-3164 & -0.0978 & $4.61 \times 10^{-5}$ & 0.003957 & hsa-miR-548m & -0.1191 & 0.000484 & 0.018165 \\
\hline hsa-miR-130b & -1.6642 & $4.72 \times 10^{-5}$ & 0.003958 & hsa-miR-3935 & 0.1933 & 0.000485 & 0.018165 \\
\hline hsa-miR-125a-5p & 1.7078 & $5.11 \times 10^{-5}$ & 0.00419 & hsa-miR-591 & -0.0909 & 0.000497 & 0.018364 \\
\hline hsa-miR-29a $a^{a}$ & 0.9139 & $5.37 \times 10^{-5}$ & 0.004299 & hsa-miR-210 & 2.3178 & 0.0005 & 0.018364 \\
\hline hsa-miR-93a & 0.2658 & $5.75 \times 10^{-5}$ & 0.004505 & hsa-miR-556-3p & -0.1154 & 0.000527 & 0.019151 \\
\hline hsa-miR-299-3p & -0.7055 & $6.01 \times 10^{-5}$ & 0.004604 & hsa-miR-548j & -0.0797 & 0.000551 & 0.01949 \\
\hline hsa-miR-4299 & -1.8101 & $6.2 \times 10^{-5}$ & 0.004646 & hsa-miR-103a & 1.0183 & 0.000553 & 0.01949 \\
\hline hsa-miR-195 a & -0.1081 & $8.79 \times 10^{-5}$ & 0.00645 & hsa-miR-4304 & 0.1412 & 0.000553 & 0.01949 \\
\hline hsa-miR-210 & 2.4038 & $8.99 \times 10^{-5}$ & 0.006465 & hsa-miR-30c-2a & -0.4667 & 0.000577 & 0.020116 \\
\hline hsa-miR-151-3p & 0.6122 & 0.000104 & 0.007232 & hsa-miR-2116 & 0.1207 & 0.000583 & 0.020146 \\
\hline hsa-miR-3616-3p & -0.4232 & 0.000105 & 0.007232 & hsa-miR-361-3p & 0.7409 & 0.00061 & 0.020859 \\
\hline hsa-miR-498 & 0.2281 & 0.000109 & 0.007391 & hsa-miR-1537 & -0.1087 & 0.000616 & 0.020877 \\
\hline
\end{tabular}

Table II. Continued. 
Table II. Continued.

\begin{tabular}{|c|c|c|c|c|c|c|c|}
\hline miRNA_ID & $\log _{2} \mathrm{FC}$ & P-value & adj.P.Val & miRNA_ID & $\log _{2} \mathrm{FC}$ & P-value & adj.P.Val \\
\hline hsa-miR-573 & -0.1069 & 0.00064 & 0.02121 & hsa-miR-619 & -0.0715 & 0.00142 & 0.031927 \\
\hline hsa-miR-3198 & -0.7624 & 0.000641 & 0.02121 & hsa-miR-30a ${ }^{a}$ & -0.9917 & 0.00144 & 0.032039 \\
\hline hsa-miR-1324 & -0.073 & 0.00065 & 0.02121 & hsa-miR-138-2 ${ }^{\mathrm{a}}$ & 0.1095 & 0.00148 & 0.032687 \\
\hline hsa-miR-99b & 1.4301 & 0.000653 & 0.02121 & hsa-miR-182 & 2.0004 & 0.00152 & 0.03353 \\
\hline hsa-miR-107 & 1.058 & 0.000656 & 0.02121 & hsa-miR-484 & 0.9217 & 0.00154 & 0.033733 \\
\hline hsa-miR-587 & -0.0948 & 0.000663 & 0.021248 & hsa-miR-425 & 0.8842 & 0.00157 & 0.03375 \\
\hline hsa-miR-586 & -0.0974 & 0.000686 & 0.021727 & hsa-miR-4310 & 0.1418 & 0.00158 & 0.03375 \\
\hline hsa-miR-325 & -0.1108 & 0.000691 & 0.021727 & hsa-miR-302c & -0.106 & 0.00158 & 0.03375 \\
\hline hsa-miR-24 & 0.9059 & 0.000705 & 0.021812 & hsa-miR-15b ${ }^{a}$ & -0.1306 & 0.00159 & 0.03375 \\
\hline hsa-miR-128 & 1.1449 & 0.000706 & 0.021812 & hsa-miR-302f & -0.0859 & 0.0016 & 0.03375 \\
\hline hsa-miR-892a & -0.1117 & 0.000713 & 0.021833 & hsa-miR-2053 & -0.1115 & 0.0016 & 0.03375 \\
\hline hsa-miR-573 & -0.109 & 0.000719 & 0.021833 & hsa-miR-4291 & 0.8359 & 0.00167 & 0.035042 \\
\hline hsa-miR-1265 & -0.0783 & 0.000727 & 0.021835 & hsa-miR-421 & 0.5925 & 0.00169 & 0.035259 \\
\hline hsa-miR-30c-1 ${ }^{\text {a }}$ & -0.3181 & 0.000731 & 0.021835 & hsa-miR-381 & -1.9681 & 0.00171 & 0.035396 \\
\hline hsa-miR-183 & 2.7044 & 0.000747 & 0.022129 & hsa-miR-3647-3p & -0.1454 & 0.00173 & 0.035724 \\
\hline hsa-miR-301a & 1.8224 & 0.000771 & 0.022642 & hsa-miR-328 & 0.803 & 0.00178 & 0.036488 \\
\hline hsa-miR-193a-3p & -1.5641 & 0.000797 & 0.0232 & hsa-miR-3620 & 0.1262 & 0.00185 & 0.037581 \\
\hline hsa-miR-125a-5p & 1.3715 & 0.000809 & 0.023366 & hsa-miR-3927 & -0.0686 & 0.0019 & 0.03843 \\
\hline hsa-miR-331-3p & 0.8881 & 0.000839 & 0.023999 & hsa-miR-92a- $2^{a}$ & -0.0791 & 0.00192 & 0.038565 \\
\hline hsa-miR-3115 & -0.0798 & 0.000845 & 0.023999 & hsa-miR-4317 & 0.3214 & 0.00193 & 0.038645 \\
\hline hsa-let-7 $\mathrm{d}^{\mathrm{a}}$ & 0.3463 & 0.000856 & 0.024136 & hsa-miR-149 & 1.1225 & 0.00196 & 0.038946 \\
\hline hsa-miR-183 & 2.6632 & 0.000864 & 0.024164 & hsa-miR-183 & 1.0302 & 0.00197 & 0.038946 \\
\hline hsa-miR-3180-5p & 0.0999 & 0.000887 & 0.024611 & hsa-miR-484 & 1.0961 & 0.00206 & 0.040621 \\
\hline hsa-miR-1539 & 0.0936 & 0.000898 & 0.024707 & hsa-miR-3198 & -0.6895 & 0.00215 & 0.041996 \\
\hline hsa-miR-1306 & 0.1827 & 0.000945 & 0.025729 & hsa-miR-190b & -0.1413 & 0.00216 & 0.041996 \\
\hline hsa-miR-3142 & -0.0824 & 0.000949 & 0.025729 & hsa-miR-553 & -0.086 & 0.0022 & 0.0425 \\
\hline hsa-miR-548d-5p & -0.1196 & 0.000977 & 0.026272 & hsa-miR-324-5p & 1.5161 & 0.00222 & 0.042666 \\
\hline hsa-miR-183 & 2.5675 & 0.001 & 0.026774 & hsa-miR-182 & 2.3243 & 0.00223 & 0.042666 \\
\hline hsa-miR-582-3p & 0.5736 & 0.00102 & 0.027098 & hsa-miR-624 ${ }^{\mathrm{a}}$ & 0.0748 & 0.00227 & 0.04329 \\
\hline hsa-miR-569 & -0.0898 & 0.00104 & 0.027306 & hsa-let-7e & 1.2111 & 0.00233 & 0.044225 \\
\hline hsa-miR-411 & -0.813 & 0.00106 & 0.027402 & hsa-miR-30a & -1.6194 & 0.00241 & 0.045407 \\
\hline hsa-miR-3668 & -0.0768 & 0.00107 & 0.027402 & hsa-miR-181b & 1.2205 & 0.00244 & 0.045689 \\
\hline hsa-miR-320b & 0.7204 & 0.00107 & 0.027402 & hsa-miR-1294 & -0.0642 & 0.00248 & 0.046233 \\
\hline hsa-miR-302f & -0.0959 & 0.00107 & 0.027402 & hsa-miR-320a & 0.4892 & 0.00251 & 0.046444 \\
\hline hsa-miR-183 ${ }^{\mathrm{a}}$ & 1.5187 & 0.00111 & 0.02783 & hsa-miR-491-5p & 0.5918 & 0.00252 & 0.046444 \\
\hline hsa-miR-93 & 0.9869 & 0.00112 & 0.02783 & hsa-miR-4293 & 0.081 & 0.00253 & 0.046485 \\
\hline hsa-miR-182 & 2.0297 & 0.00113 & 0.02783 & hsa-miR-345 & 0.2807 & 0.00259 & 0.047307 \\
\hline hsa-miR-4317 & 0.7724 & 0.00113 & 0.02783 & hsa-miR-563 & 0.0935 & 0.00268 & 0.048439 \\
\hline hsa-miR-544 & -0.1508 & 0.00114 & 0.02783 & hsa-miR-219-1-3p & -0.0921 & 0.00268 & 0.048439 \\
\hline hsa-miR-1179 & -0.074 & 0.00114 & 0.02783 & hsa-miR-3924 & -0.0974 & 0.0027 & 0.048517 \\
\hline hsa-miR-1243 & -0.0774 & 0.00116 & 0.028001 & hsa-miR-367 ${ }^{\mathrm{a}}$ & -0.0887 & 0.0028 & 0.050028 \\
\hline hcmv-miR-UL36 & -0.0762 & 0.00116 & 0.028001 & ebv-miR-BART10 & -0.1116 & 0.00282 & 0.050028 \\
\hline hsa-miR-30a $a^{a}$ & -1.209 & 0.00117 & 0.028137 & hsa-miR-1284 & -0.0713 & 0.00283 & 0.050028 \\
\hline hsa-miR-1179 & -0.073 & 0.00123 & 0.029223 & hsa-miR-1291 & 0.1029 & 0.00286 & 0.05032 \\
\hline hsa-miR-3140-3p & -0.1157 & 0.00124 & 0.029223 & hsa-miR-608 & -0.057 & 0.00298 & 0.052183 \\
\hline hsa-miR-1286 & 0.0726 & 0.00124 & 0.029223 & hsa-miR-425 & 0.8292 & 0.003 & 0.052407 \\
\hline hsa-miR-563 & -0.1156 & 0.00125 & 0.029223 & hsa-miR-4291 & 0.9583 & 0.00315 & 0.054597 \\
\hline hsa-miR-30e & -0.7409 & 0.00127 & 0.029223 & hsa-miR-19b-2a & -0.1085 & 0.00316 & 0.054597 \\
\hline ebv-miR-BART8 & -0.0971 & 0.00127 & 0.029223 & hsa-miR-19b-1 ${ }^{a}$ & 0.1263 & 0.00319 & 0.054855 \\
\hline hsa-miR-4307 & -0.1001 & 0.00128 & 0.029223 & hsa-miR-548u & -0.0593 & 0.00328 & 0.056055 \\
\hline hsa-miR-3158-3p & -0.054 & 0.00141 & 0.031927 & hsv2-miR-H22 & -1.2156 & 0.0033 & 0.056055 \\
\hline hsa-miR-520h & -0.1088 & 0.00142 & 0.031927 & hsa-miR-374c & 0.1584 & 0.00331 & 0.056055 \\
\hline
\end{tabular}

Table II. Continued. 
Table II. Continued.

\begin{tabular}{|c|c|c|c|}
\hline miRNA_ID & $\log _{2} \mathrm{FC}$ & P-value & adj.P.Val \\
\hline hsa-miR-219-5p & -0.5987 & 0.00333 & 0.056055 \\
\hline hsa-miR-29c ${ }^{a}$ & 1.0172 & 0.00338 & 0.056676 \\
\hline hsa-miR-493 ${ }^{a}$ & -0.7182 & 0.00339 & 0.056676 \\
\hline hsa-miR-302a $a^{a}$ & 0.0797 & 0.0035 & 0.057987 \\
\hline hsa-miR-130b & -0.097 & 0.00351 & 0.057987 \\
\hline hsa-miR-651 & -0.079 & 0.00352 & 0.057987 \\
\hline hiv1-miR-TAR-3p & 0.361 & 0.00357 & 0.058286 \\
\hline hsa-miR-24 & 0.6936 & 0.00357 & 0.058286 \\
\hline hsa-let-7d $\mathrm{d}^{\mathrm{a}}$ & 0.2023 & 0.00363 & 0.058837 \\
\hline hsa-miR-140-5p & 1.0041 & 0.00364 & 0.058837 \\
\hline hsa-miR-548b-3p & -0.0828 & 0.00368 & 0.059172 \\
\hline hsa-miR-3154 & 0.31 & 0.00371 & 0.059172 \\
\hline hsa-miR-324-5p & 1.3843 & 0.00372 & 0.059172 \\
\hline hsa-miR-33a & 0.5461 & 0.00374 & 0.059172 \\
\hline hsa-miR-154 & -1.5917 & 0.00375 & 0.059172 \\
\hline hsa-miR-107 & 0.966 & 0.00381 & 0.059939 \\
\hline hsv2-miR-H22 & -1.1749 & 0.00393 & 0.061263 \\
\hline hsa-miR-3649 & -0.068 & 0.00395 & 0.061263 \\
\hline hsa-miR-182a & 0.8636 & 0.00396 & 0.061263 \\
\hline hsa-miR-501-3p & 0.3569 & 0.00396 & 0.061263 \\
\hline hsa-miR-449b & -0.0656 & 0.0041 & 0.062902 \\
\hline hsa-miR-3942-5p & -0.0792 & 0.00412 & 0.062902 \\
\hline hsa-let-7e & 1.2761 & 0.00412 & 0.062902 \\
\hline hcmv-miR-UL22A ${ }^{a}$ & -0.0787 & 0.00421 & 0.063705 \\
\hline hsa-miR-3684 & -0.0467 & 0.00426 & 0.063705 \\
\hline hsa-miR-585 & -0.0906 & 0.00427 & 0.063705 \\
\hline hsa-miR-3606 & -0.0616 & 0.00427 & 0.063705 \\
\hline hsa-miR-191 & 0.2472 & 0.00428 & 0.063705 \\
\hline hsa-miR-18b ${ }^{a}$ & -0.0939 & 0.0043 & 0.063705 \\
\hline hsa-miR-4309 & -0.056 & 0.00432 & 0.063705 \\
\hline hcmv-miR-UL22A & 0.0758 & 0.00432 & 0.063705 \\
\hline hsa-miR-320c & 0.6075 & 0.00444 & 0.06516 \\
\hline hsa-miR-942 & -0.1076 & 0.00455 & 0.066499 \\
\hline hsa-miR-3190 & 0.1172 & 0.00458 & 0.066689 \\
\hline hsa-miR-374a $a^{a}$ & -0.0927 & 0.00461 & 0.066754 \\
\hline hsa-miR-3675-3p & 0.0965 & 0.00462 & 0.066754 \\
\hline hsa-miR-374c & 0.6226 & 0.00467 & 0.067088 \\
\hline hsa-miR-3152-3p & -0.0574 & 0.00474 & 0.067654 \\
\hline hsa-miR-3189-3p & 0.1598 & 0.00474 & 0.067654 \\
\hline hsa-miR-29a & 0.6544 & 0.00482 & 0.067818 \\
\hline hsa-miR-892a & -0.0808 & 0.00482 & 0.067818 \\
\hline hsa-miR-2355-5p & 0.358 & 0.00483 & 0.067818 \\
\hline
\end{tabular}

${ }^{\mathrm{a}} \mathrm{miR}$ with lower expression levels; FC, fold change; miR, microRNA.

provided us with the expression of thousands of genes in the human genome, and numerous studies have already enabled certain predictions to be made using these data (34-36). The aim is to identify novel targets through such techniques, in order to guide the treatment of pancreatic cancer and improve the efficiency of early diagnosis.
The advent of miRNA research has opened up novel avenues for understanding the post-transcriptional level of gene regulation. The discovery of dysregulated miRNAs in a variety of cancer types has led numerous researchers to study the use of miRNAs as potential biomarkers for early detection, as well as therapeutic agents for the treatment of cancer (11-12). miRNAs are short (19-24 nucleotides) non-coding RNA molecules, which have emerged as promising prognostic, diagnostic and therapeutic tools that may be targeted in the fight against pancreatic cancer (37-40).

In the present study, the miRNA sequencing profiles of 185 pancreatic tumor samples from the TCGA database have been presented. GEO datasets were obtained through a reading of the literature to explore which one would be of the greatest benefit for analysis. The most recent datasets were selected and contained $>30$ samples. In the majority of recently published studies, the TCGA and GEO databases have usually been selected to expand the sample range at the beginning of the process of screening data, and other data to accurately quantify the results in subsequent analyses have not subsequently been introduced. However, the present study factored in a novel approach: The continuous introduction of GEO datasets to narrow the scope of the filter, in an attempt to render the results more accurate.

By retrieving literature in PubMed, a total of 14 articles were identified that related of hsa-miR-3613-5p, 6 of which exhibited meaningful results. hsa-miR-3613 was a newly identified miRNA, which has been found to be related to gastric cancer (41). Chen et al (42) demonstrated that hsa-miR-3613 is upregulated in drug-resistant breast cancer cell lines through Affymetrix GeneChip miRNA 4.0 array. However, the experiments suggested that it was not able to serve as a potential source for biomarker detection or as an optimal chemotherapeutic choice for patients with breast cancer. Hsa-miR-3613 was revealed in a study by Chong et al (43) to be one of the most markedly downregulated miRNAs in recurrent epithelial ovarian cancer (EOC), and may be regarded as a biomarker for the prediction of recurrence in EOC. Castro-Magdonel et al (44) demonstrated that the presence of hsa-miR-3613 is critical for tumor suppression in retinoblastoma. In addition, a further study in the published literature has revealed that hsa-miR-3613 may be associated with leukemia/small lymphocytic lymphoma (45). Hsa-miR-3613 was also demonstrated to be highly expressed in gluteofemoral compared with subcutaneous adipocytes (46). To the best of our knowledge, however, no articles have been published that have explored the association between hsa-miR-3613 and pancreatic cancer. Therefore, the present study aimed to investigate this association via high-throughput sequencing analyses.

In addition, genes located downstream of hsa-miR-3613 were also analyzed. The two most recently updated target prediction databases were selected to predict the target(s) of hsa-miR-3613, and KEGG and GO analyses of the target genes were performed to probe for points of intersection.

Previously published studies have revealed the p53 signaling pathway is an important pathway that is closely associated with cancer development (47-50), and mutations in tumor protein 53 (TP53) elicited changes in the expression 

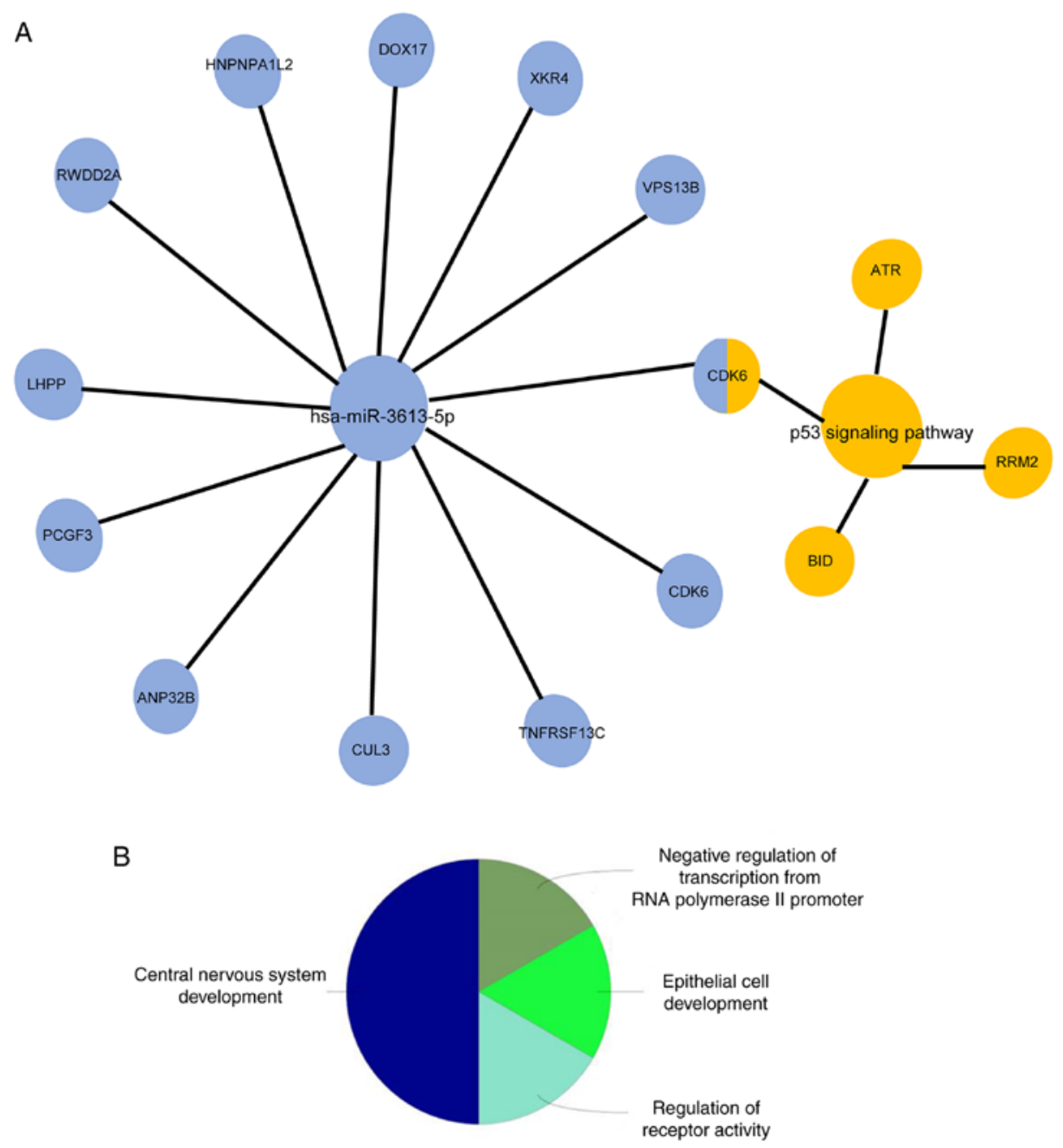

Figure 5. Functional analyses of the hsa-miR-3613-5p target genes. (A) KEGG pathway network of hsa-miR-3613-5p-overlapping target genes. The diamond represents hsa-miR-3613-5p, whereas the circles represent pathways enriched in overlapping genes. Circles in red denote the overlapping genes. (B) Significantly enriched GO biological processes $(\mathrm{P}<0.05)$ are presented in the pie chart. KEGG, Kyoto Encyclopedia of Genes and Genomes; miR, microRNA; GO, Gene Ontology.

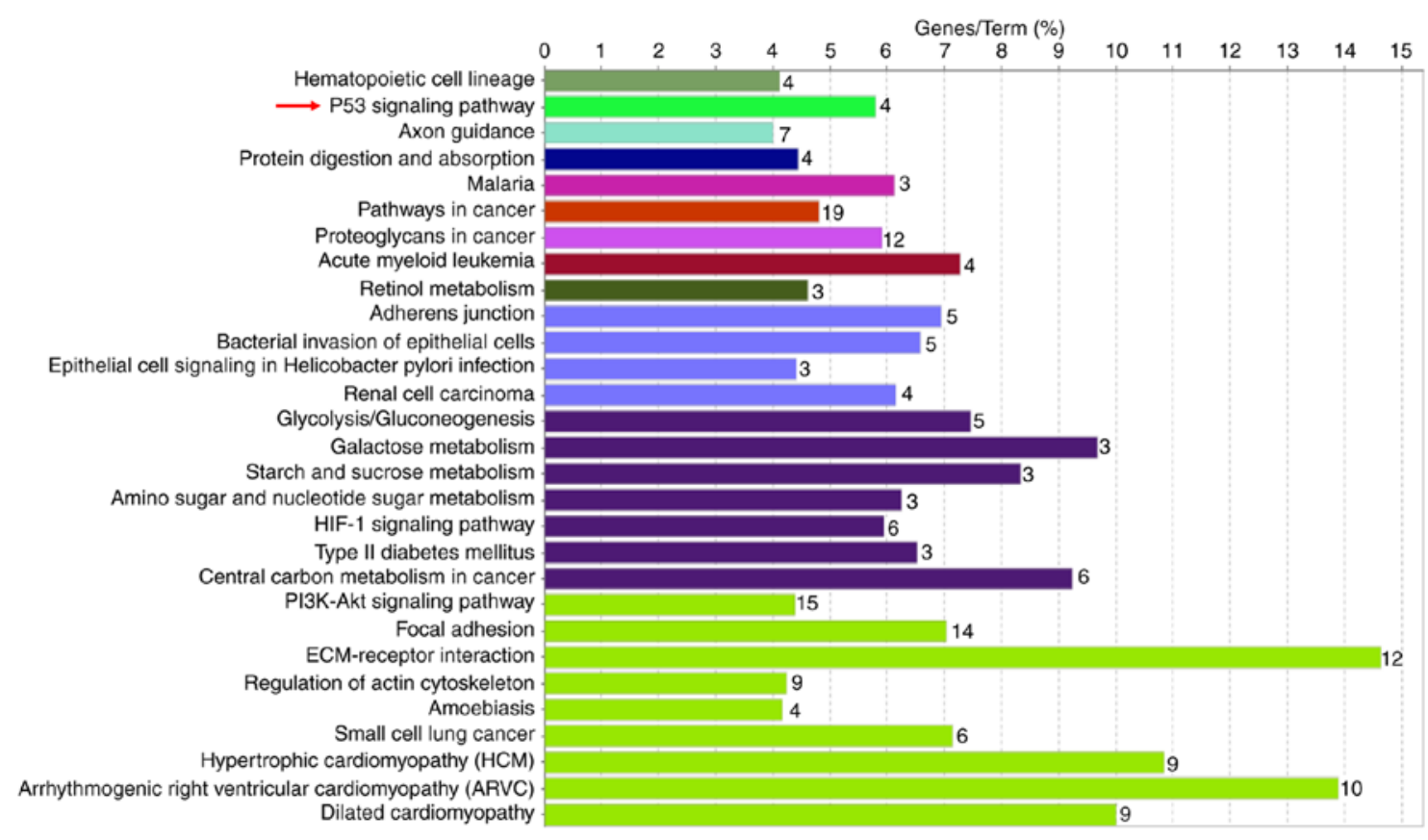

Figure 6. Functional analysis of the top 250 genes in GSE62452. The significantly enriched KEGG pathways are shown in the bar chart. Red arrow indicates the location of 533 signaling pathway. KEGG, Kyoto Encyclopedia of Genes and Genomes. 

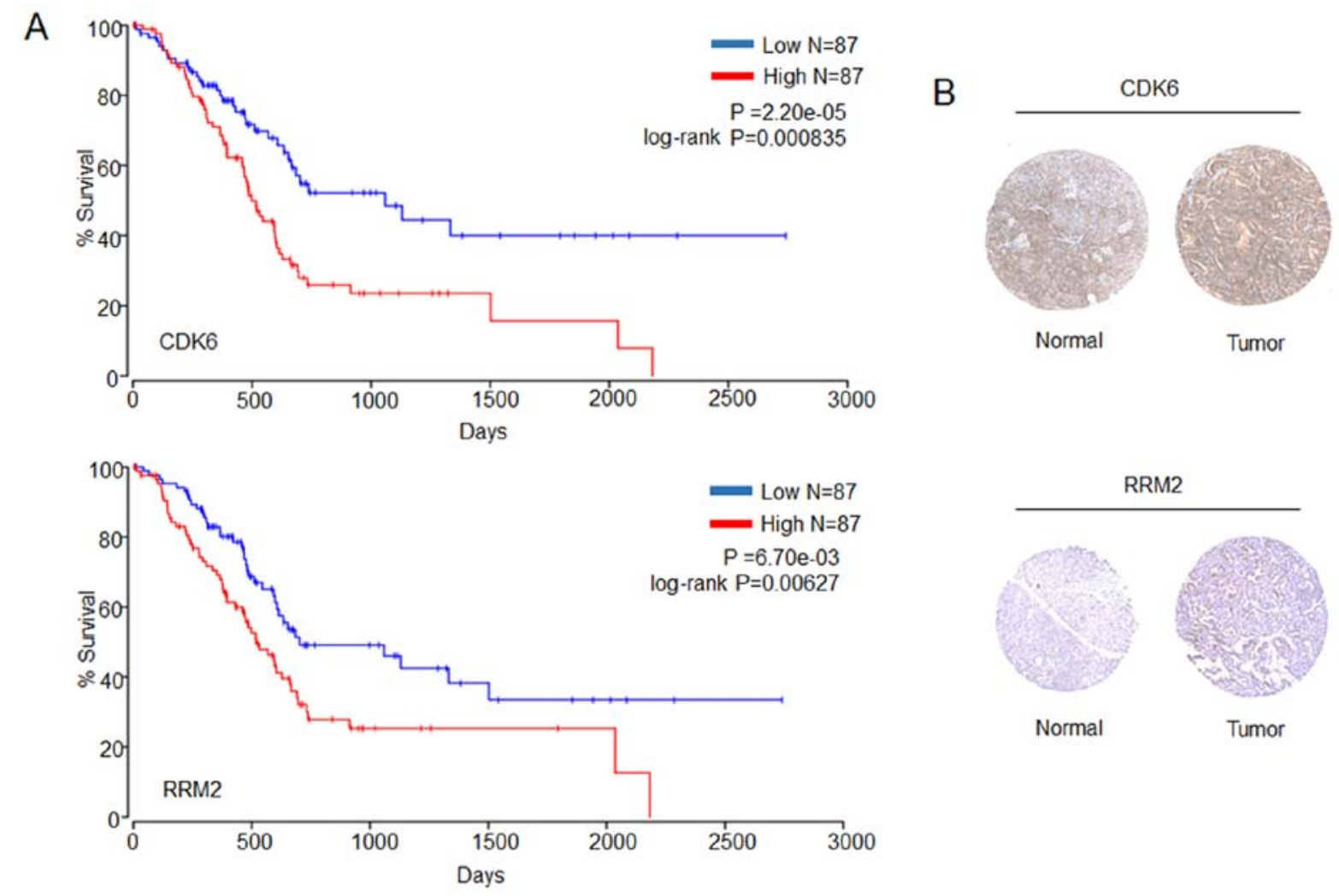

Figure 7. Survival analysis and gene expression comparisons in non-cancer and cancer tissues of the CDK6 and RRM2 genes. (A) Overall survival analysis using OncoLnc. (B) Expression of the CDK6 and RRM2 genes in non-cancer and cancer tissues according to immunohistochemistry experiments. CDK6, cyclin-dependent kinase 6; RRM2, ribonucleoside-diphosphate reductase subunit M2.

of numerous other genes (51-53). Two reviews related to pancreatic cancer attracted attention. Makohon-Moore and Iacobuzio-Donahue (54) reported that genomic features are closely associated with the pathogenesis of pancreatic cancer, and TP53 undergoes somatic mutations in up to $85 \%$ of pancreatic cancers. Another review, written by Rachagani et al (37), summarized the relationship between miRNAs and tumor suppressor genes (including p53, p16 and SMAD4) in the pathogenesis, diagnosis and therapy of pancreatic cancer. This study concluded that p53 not only has the potential to regulate miRNA expression, but in turn miRNA can also regulate p53 expression. Additionally, the prognostic value of genes in the p53 signaling pathway were evaluated during the current analysis, revealing that the genes for CDK6 and RRM2 are closely associated with prognostic value.

Taken together, through comprehensive bioinformatics analysis, the results presented in the current study identified a prognostic miRNA involved in the progression of pancreatic cancer. These findings may lead to the identification of a number of key miRNAs, genes and pathways for future investigation into the mechanisms and biomarkers of pancreatic cancer. However, the present study did have certain limitations. The main limitation of the present study was due to the retrospective nature of the analysis. At the same time, the small sample size reduced the statistical power, and the limited number of samples expressing hsa-miR-3613 should also be considered as a limiting factor in data analysis. In addition, the data presented in the current study could not fully explain why hsa-miR-3613 was expressed at a high level in pancreatic cancer and resulted in an improved overall prognosis. Further studies using cancer cell lines and animal models should be undertaken in the future to gain additional mechanistic insight into the implications of hsa-miR-3613 expression in disease progression.

\section{Acknowledgements}

Not applicable.

\section{Funding}

The present study was financially supported by the National Natural Science Foundation of China (grant no. 81872156).

\section{Availability of data and materials}

The miRNA sequencing data of pancreatic cancer are available in FireBrowse (version 0.4.13; http://firebrowse.org/). The datasets of miRNA expression profiles for pancreatic cancer (GSE43796) and gene expression profiles for pancreatic cancer (GSE62452) are available in the GEO on the NCBI website (http://www.ncbi.nlm.nih.gov/geo).

\section{Authors' contributions}

JM and SS carried out the design of this study, performed the statistical analysis and drafted the manuscript. CS, NiL, 
$\mathrm{NaL}$ and LX promoted the development of this project, and interpreted the data regarding the functional and pathway enrichment and PPI network construction. TY and YL helped to edit the manuscript, and downloaded datasets from the GEO and interpreted the primary data regarding pancreatic cancer. ML participated in the study design and coordination and also helped to edit the manuscript. All authors read and approved the final manuscript.

\section{Ethics approval and consent to participate}

Not applicable.

\section{Patient consent for publication}

Not applicable.

\section{Competing interests}

The authors declare that they have no competing interests.

\section{References}

1. Shimura T, Shibata M, Gonda K, Kofunato Y, Okada R, Ishigame T, Kimura T, Kenjo A, Kono K and Marubashi S: Significance of circulating galectin-3 in patients with pancreatobiliary cancer. Anticancer Res 37: 4979-4986, 2017.

2. Ohya A, Yamanoi K, Shimojo H, Fujii C and Nakayama J: Gastric gland mucin-specific o-glycan expression decreases with tumor progression from precursor lesions to pancreatic cancer. Cancer Sci 108: 1897-1902, 2017.

3. Park J, Choi Y, Namkung J, Yi SG, Kim H, Yu J, Kim Y, Kwon MS, Kwon W, Oh DY, et al: Diagnostic performance enhancement of pancreatic cancer using proteomic multimarker panel. Oncotarget 8: 93117-93130, 2017.

4. Kwon HM, Kang EJ, Kang K, Kim SD, Yang K and Yi JM: Combinatorial effects of an epigenetic inhibitor and ionizing radiation contribute to targeted elimination of pancreatic cancer stem cell. Oncotarget 8: 89005-89020, 2017.

5. Han T, Zhuo M, Hu H, Jiao F and Wang LW: Synergistic effects of the combination of 5-Aza-CdR and suberoylanilide hydroxamic acid on the anticancer property of pancreatic cancer. Oncol Rep 39: 264-270, 2018.

6. Brooks J, Fleischmann-Mundt B, Woller N, Niemann J, Ribback S, Peters K, Demir IE, Armbrecht N, Ceyhan GO, Manns MP, et al: Perioperative, spatiotemporally coordinated activation of $\mathrm{T}$ and NK cells prevents recurrence of pancreatic cancer. Cancer Res 78: 475-488, 2017.

7. Mirkin KA, Hollenbeak CS and Wong J: Greater lymph node retrieval and lymph node ratio impacts survival in resected pancreatic cancer. J Surg Res 220: 12-24, 2017.

8. Khan MA, Azim S,Zubair H, Bhardwaj A, Patel GK, Khushman M, Singh S and Singh AP: Molecular drivers of pancreatic cancer pathogenesis: Looking inward to move forward. Int J Mol Sci 18: E779, 2017.

9. Du YX, Liu ZW, You L, Wu WM and Zhao YP: Advances in understanding the molecular mechanism of pancreatic cancer metastasis. Hepatobiliary Pancreat Dis Int 15: 361-370, 2016.

10. Chen L and Kang C: miRNA interventions serve as 'magic bullets' in the reversal of glioblastoma hallmarks. Oncotarget 6 : 38628-38642, 2015.

11. Liu W, Ma R and Yuan Y: Post-transcriptional regulation of genes related to biological behaviors of gastric cancer by long noncoding RNAs and microRNAs. J Cancer 8: 4141-4154, 2017.

12. Shirafkan N, Mansoori B, Mohammadi A, Shomali N, Ghasbi M and Baradaran B: MicroRNAs as novel biomarkers for colorectal cancer: New outlooks. Biomed Pharmacother 97: 1319-1330, 2017.

13. Kim VN, Han J and Siomi MC: Biogenesis of small RNAs in animals. Nat Rev Mol Cell Biol 10: 126-139, 2009.
14. Huang L, Cai JL, Huang PZ, Kang L, Huang MJ, Wang L and Wang JP: miR19b-3p promotes the growth and metastasis of colorectal cancer via directly targeting ITGB8. Am J Cancer Res 7: 1996-2008, 2017.

15. Long M, Zhan M, Xu S, Yang R, Chen W, Zhang S, Shi Y, He Q, Mohan M, Liu Q and Wang J: miR-92b-3p acts as a tumor suppressor by targeting Gabra3 in pancreatic cancer. Mol Cancer 16: 167, 2017.

16. Bayraktar R, Van Roosbroeck K and Calin GA: Cell-to-cell communication: Micrornas as hormones. Mol Oncol 11: 1673-1686, 2017.

17. Fujiwara T, Uotani K, Yoshida A, Morita T, Nezu Y, Kobayashi E, Yoshida A,Uehara T, Omori T,Sugiu K, et al: Clinical significance of circulating miR-25-3p as a novel diagnostic and prognostic biomarker in osteosarcoma. Oncotarget 8: 33375-33392, 2017.

18. Yu Y, Zuo J, Tan Q, Zar Thin K, Li P, Zhu M, Yu M, Fu Z, Liang $\mathrm{C}$ and Tu J: Plasma miR-92a-2 as a biomarker for small cell lung cancer. Cancer Biomark 18: 319-327, 2017.

19. Schwarzenbach $\mathrm{H}$ : Clinical relevance of circulating, cell-free and exosomal microRNAs in plasma and serum of breast cancer patients. Oncol Res Treat 40: 423-429, 2017.

20. Deng W, Wang Y, Liu Z, Cheng H and Xue Y: HemI: A toolkit for illustrating heatmaps. PLoS One 9: e111988, 2014.

21. Yang S, He P, Wang J, Schetter A, Tang W, Funamizu N, Yanaga K, Uwagawa T, Satoskar AR, Gaedcke J, et al: A novel MIF signaling pathway drives the malignant character of pancreatic cancer by targeting NR3C2. Cancer Res 76: 3838-3850, 2016.

22. Park M, Kim M, Hwang D, Park M, Kim WK, Kim SK, Shin J, Park ES, Kang CM, Paik YK and Kim H: Characterization of gene expression and activated signaling pathways in solid-pseudopapillary neoplasm of pancreas. Mod Pathol 27: 580-593, 2014.

23. Anaya J: OncoLnc: Linking TCGA survival data to mRNAs, miRNAs, and lncRNAs. PeerJ Computer Science 2: e67, 2016.

24. Agarwal V, Bell GW, Nam JW and Bartel DP: Predicting effective microRNA target sites in mammalian mRNAs. Elife 4: 7554, 2015.

25. Paraskevopoulou MD, Georgakilas G, Kostoulas N, Vlachos IS, Vergoulis T, Reczko M, Filippidis C, Dalamagas T and Hatzigeorgiou AG: DIANA-microT web server v5.0: Service integration into miRNA functional analysis workflows. Nucleic Acids Res 41: W169-W173, 2014.

26. Reczko M, Maragkakis M, Alexiou P, Grosse I and Hatzigeorgiou AG: Functional microRNA targets in protein coding sequences. Bioinformatics 28: 771-776, 2012.

27. Shannon P, Ozier O, Baliga NS, Wang JT, Ramage D, Amin N, Schwikowski B, Ideker T and Markiel A: Cytoscape: A software environment for integrated models of biomolecular interaction networks. Genome Res 13: 2498-2504, 2003.

28. Pontén F, Jirström K and Uhlen M: The human protein atlas-a tool for pathology. J Pathol 216: 387-393, 2008.

29. Karmakar S, Kaushik G, Nimmakayala R, Rachagani S, Ponnusamy MP and Batra SK: MicroRNA regulation of K-ras in pancreatic cancer and opportunities for therapeutic intervention. Semin Cancer Biol 54: 63-71, 2017.

30. Lu Z, Lai ZQ, Leung AWN, Leung PS, Li ZS and Lin ZX: Exploring brusatol as a new anti-pancreatic cancer adjuvant: Biological evaluation and mechanistic studies. Oncotarget 8: 84974-84985, 2017.

31. Choi M, Bien H, Mofunanya A and Powers S: Challenges in ras therapeutics in pancreatic cancer. Semin Cancer Biol 54: 101-108, 2017.

32. Halbrook CJ and Lyssiotis CA: Employing metabolism to improve the diagnosis and treatment of pancreatic cancer. Cancer Cell 31: 5-19, 2017.

33. Wang L and Xie K: Nitric oxide and pancreatic cancer pathogenesis, prevention, and treatment. Curr Pharm Des 16: 421-427, 2010.

34. Chen WJ, Tang RX, He RQ, Li DY, Liang L, Zeng JH, Hu XH, Ma J,Li SK and Chen G: Clinical roles of the aberrantly expressed lncRNAs in lung squamous cell carcinoma: A study based on RNA-sequencing and microarray data mining. Oncotarget 8: 61282-61304, 2017.

35. Chen Y, Teng L, Liu W, Cao Y, Ding D, Wang W, Chen H, Li C and An R: Identification of biological targets of therapeutic intervention for clear cell renal cell carcinoma based on bioinformatics approach. Cancer Cell Int 16: 16, 2016.

36. Liang B, Li Y and Wang T: A three miRNAs signature predicts survival in cervical cancer using bioinformatics analysis. Sci Rep 7: 5624, 2017. 
37. Rachagani S, Macha MA, Heimann N, Seshacharyulu $P$, Haridas D, Chugh S and Batra SK: Clinical implications of miRNAs in the pathogenesis, diagnosis and therapy of pancreatic cancer. Adv Drug Delivy Rev 81: 16-33, 2015.

38. Chitkara D, Mittal A and Mahato RI: miRNAs in pancreatic cancer: Therapeutic potential, delivery challenges and strategies. Adv Drug Deliv Rev 81: 34-52, 2015.

39. Li L, Li Z, Kong X, Xie D, Jia Z, Jiang W, Cui J, Du Y, Wei D, Huang $S$ and Xie K: Down-regulation of microRNA-494 via loss of SMAD4 increases FOXM1 and $\beta$-catenin signaling in pancreatic ductal adenocarcinoma cells. Gastroenterology 147: 485-497, 2014.

40. Schultz NA, Dehlendorff C, Jensen BV, Bjerregaard JK, Nielsen KR, Bojesen SE, Calatayud D, Nielsen SE, Yilmaz M, Holländer $\mathrm{NH}$, et al: MicroR NA biomarkers in whole blood for detection of pancreatic cancer. JAMA 311: 392-404, 2014.

41. Bibi F, Naseer MI, Alvi SA, Yasir M, Jiman-Fatani AA, Sawan A, Abuzenadah AM, Al-Qahtani MH and Azhar EI: MicroRNA analysis of gastric cancer patients from Saudi Arabian population. BMC Genomics 17: 751, 2016.

42. Chen X, Lu P, Wang DD, Yang SJ, Wu Y, Shen HY, Zhong SL, Zhao JH and Tang JH: The role of miRNAs in drug resistance and prognosis of breast cancer formalin-fixed paraffin-embedded tissues. Gene 595: 221-226, 2016

43. Chong GO, Jeon HS, Han HS, Son JW, Lee YH, Hong DG, Lee YS and Cho YL: Differential microrna expression profiles in primary and recurrent epithelial ovarian cancer. Anticancer Res 35: 2611-2617, 2015.

44. Castro-Magdonel BE, Orjuela M, Camacho J, GarcíaChéquer AJ, Cabrera-Muñoz L, Sadowinski-Pine S, Durán-Figueroa N, Orozco-Romero MJ, Velázquez-Wong AC, Hernández-Ángeles A, et al: miRNome landscape analysis reveals a 30 miRNA core in retinoblastoma. BMC Cancer 17: 458, 2017.

45. Grygalewicz B, Woroniecka R, Rygier J, Borkowska K, Rzepecka I, Łukasik M, Budziłowska A, Rymkiewicz G, Błachnio K, Nowakowska B, et al: Monoallelic and biallelic deletions of $13 \mathrm{q} 14$ in a group of CLL/SLL patients investigated by CGH haematological cancer and SNP array $(8 \times 60 \mathrm{~K})$. Mol Cytogenet 9: 1,2016.
46. Tsiloulis T, Pike J, Powell D, Rossello FJ, Canny BJ, Meex RC and Watt MJ: Impact of endurance exercise training on adipocyte microRNA expression in overweight men. FASEB J 31: 161-171, 2017.

47. Liu K, Zhao X, Gu J, Wu J, Zhang H and Li Y: Effects of $12 \mathrm{C} 6+$ heavy ion beam irradiation on the p53 signaling pathway in HepG2 liver cancer cells. Acta Biochim Biophys Sin (Shanghai) 49: 989-998, 2017.

48. Song D, Zhao J, Su C, Jiang Y and Hou J: Etoposide induced NMI promotes cell apoptosis by activating the ARF-p53 signaling pathway in lung carcinoma. Biochem Biophys Res Commun 495: 368-374, 2017.

49. Wu Z, Zhu X, Xu W, Zhang Y, Chen L, Qiu F, Zhang B, Wu L, Peng $\mathrm{Z}$ and Tang $\mathrm{H}$ : Up-regulation of CIT promotes the growth of colon cancer cells. Oncotarget 8: 71954-71964, 2017.

50. Mello SS, Valente LJ, Raj N, Seoane JA, Flowers BM, McClendon J, Bieging-Rolett KT, Lee J, Ivanochko D, Kozak MM, et al: A p53 super-tumor suppressor reveals a tumor suppressive p53-Ptpn14-Yap axis in pancreatic cancer. Cancer Cell 32: 460-473, 2017.

51. Morita K, Noura M, Tokushige C, Maeda S, Kiyose H, Kashiwazaki G, Taniguchi J, Bando T, Yoshida K, Ozaki T, et al: Autonomous feedback loop of RUNX1-p53-CBFB in acute myeloid leukemia cells. Sci Rep 7: 16604, 2017.

52. Chen H, Qian J, Werner S, Cuk K, Knebel P and Brenner H: Development and validation of a panel of five proteins as blood biomarkers for early detection of colorectal cancer. Clin Epidemiol 9: 517-526, 2017

53. Masetti M, Acquaviva G, Visani M, Tallini G, Fornelli A, Ragazzi M, Vasuri F, Grifoni D, Di Giacomo S, Fiorino S, et al: Long-term survivors of pancreatic adenocarcinoma show low rates of genetic alterations in KRAS, TP53 and SMAD4. Cancer Biomark 6: 323-334, 2017.

54. Makohon-Moore A and Iacobuzio-Donahue CA: Pancreatic cancer biology and genetics from an evolutionary perspective. Nat Rev Cancer 16: 553-565, 2016.

This work is licensed under a Creative Commons Attribution-NonCommercial-NoDerivatives 4.0 International (CC BY-NC-ND 4.0) License. 